\title{
Load Aspect Recognition Based Data Compression in Smart Meters
}

\author{
K. Anupallav ${ }^{1}$, D. Selva Mary ${ }^{2}$, M. Bincy Immaculate ${ }^{3}$, U. Logeswari ${ }^{4}$, S. Uma $^{5}$ \\ Department of Information Technology, Panimalar Engineering College, Chennai ${ }^{1,2,3,4}$ \\ Guide, Department of Information Technology, Panimalar Engineering College, Chennai ${ }^{5}$
}

\begin{abstract}
Power line carrier communication has as of late pulled in the consideration of vitality organizations as a helpful and common innovation for building the advanced prepaid metering infrastructure and to balance the electricity without fluctuation. The voltage fluctuation is also reduced. The customer and the system can be interacted without the help of EB officers. This system particularly focus on the activities of power line communication technology and provide the electricity to the customer without any fluctuation till the maximum level of electricity consumption is reached. A person need not go to EB station for billing and payment.
\end{abstract}

Keywords: Power line, EB station, Load Aspect Recognition, Data Compression, Smart Meters.

\section{INTRODUCTION}

The traditional manual metering system brings disruption to users, and also wastes a lot of human energy and cost. With increasing electricity network the workload of electricity consumption is becoming high and the distribution management is also not easy. So, to avoid this issues the power line carrier communication (PLCC) transmission is introduced which can replace the usage of two wires, it can carry both data and power simultaneously. It also avoids voltage fluctuation, that can be made possible by providing the equal distribution of electricity till the maximum consumption of energy is reached and when there is more need to a particular house the fluctuation occurs, this is avoided by systematic use of the electricity fluctuation to the same house so that the nearer house are not affected and simultaneously the EB station gets a notification through a buzzer and provides extra electricity to that particular area, so this system benefits people and reduces man power. This Prepaid metering system gets an amount from RFID tag and reads it continuously which also deduce the amount accordingly for each unit of energy consumption, when the amount is reached half of the amount and also when the amount is zero it indicates the buzzer so ,that the user may be aware of the units of current consumed and can use accordingly and recharge respectively.

The major issues is that the occurrence of voltage fluctuation. This is reduced by equally distributing the electricity to all houses first and then if a particular house is consuming the energy more than the maximum level then there occurs a fluctuation to the near-by houses since the current is consumed more by one particular house, inorder to avoid this situation when there occurs fluctuation it is made systematic use to the same house which consumes more energy and then the issue is noted by the EB station so that he is then provided by extra electricity by allowing that particular house.

\section{RELATED WORK}

Automatic reading system based on automatic alignment control for pointer meter (2014)

Wuhan Univ., Wuhan, China; Yanjun Fang; Yao He; Fei Yang

In perspective of the imperfections of acknowledgment estimation of the current high exactness pointer meter, we built up an arrangement of arrangement of programmed perusing in light of programmed arrangement control for pointer meter. We changed the current working table, added to an arrangement of arrangement of camera programmed arrangement, and achieved the camera pointed consequently in view of machine vision. With respect to esteem, we enhanced the customary separation technique, in the interim, extended the scale stamp and pointer through changing over polar arrange, and changed edge strategy in unique picture into the separation strategy in polar facilitate plane, so it is more precise for acknowledgment esteem according to the position relationship amongst pointer and neighbouring scale marks.

The wireless automatic meter reading and control system based on STC12C5A60S2

Zhenjiang Cai ; Jiejing Li ; Meng Zhang

For meeting the practical needs of the rapid development in the electric power system, the automatic meter reading system is designed in this paper. The micro-controller STC12C5A60S2 is used as the core-component. The 485 circuit which is made up of the half duplex (HD) transceiver SN65LBC184 is used for data acquisition. The whole circuit is controlled by relay which connected with optical coupler in the system. After processing the data of electric meter collected by the acquisition module, it will be stored in the FERAM FM25256. Then the data could be sent to the power supply departments or customers periodically or according to the need. When the data is 
abnormal (deficiency of electric quantity, the abnormal current or voltage), the control circuit should be powered down.

Design of a Wireless ARM-Based Automatic Meter Reading and Control System (2004)

Chih-Hung Wu, Shun-Chien Chang and Yu-Wei Huang

This paper executes a remote ARM-based programmed meter perusing and control framework (WAMRCS) for dissemination robotization. The WAMRCS is planned in light of a32-bit ARM chip to manage control information preparing and transfer control.

So as to give a financially savvy, remote, constantly associated, two-way information connect between service organization and WAMRCS, the WAMRCS sends data of utility utilization, control quality and blackout alert to service organization by means of GPRS system. Contrasted and simple utility meter perusing by labour, WAMRCS is more precise, solid, practical, brisk and free from man-made mistake

\section{EXISTING WORK}

In existing system, the possibility to use the networks for the grid monitoring system has been explored. It is validated through a dedicated experimental set-up. Each smart meters is connected to the MDC by means of a performing broadband power line communication network.

The major drawback of existing system is leads to the current loss if the person is not there in home and it is low efficiency, and the person cannot go to EB office if the power goes in the night. The another drawback of existing system is that the grid infrastructure assigns power equally to all clusters and when one particular node consumes more power that are actually unused by other nodes, when that node need power back it is unable to provide.

\section{PROPOSED WORK}

In this proposed system we going to implement that $\mathrm{A}$ PLCC Based Reliable and Efficient Power Metering System for Energy Managemen tPower metering system will monitor the consumed power in particular home and transmitted via PLCC. The bill payment section will be take place in home itself.

RFID reader will detect the smart card and amount selection via keypad. Controller will check remaining amount continually, If balance is below certain level buzzer will be turn on. When the person is aware about the consumed electricity then there may be a chance of sustainable usage and the recharge can be done whenever required. It avoids low voltage (fluctuation) and balances the supply which is a basic need consumption and low voltage is directed to the high power consumed point. So, that the distributed power supply can be used efficiently.

\section{ARCHITECTURE DIAGRAM}

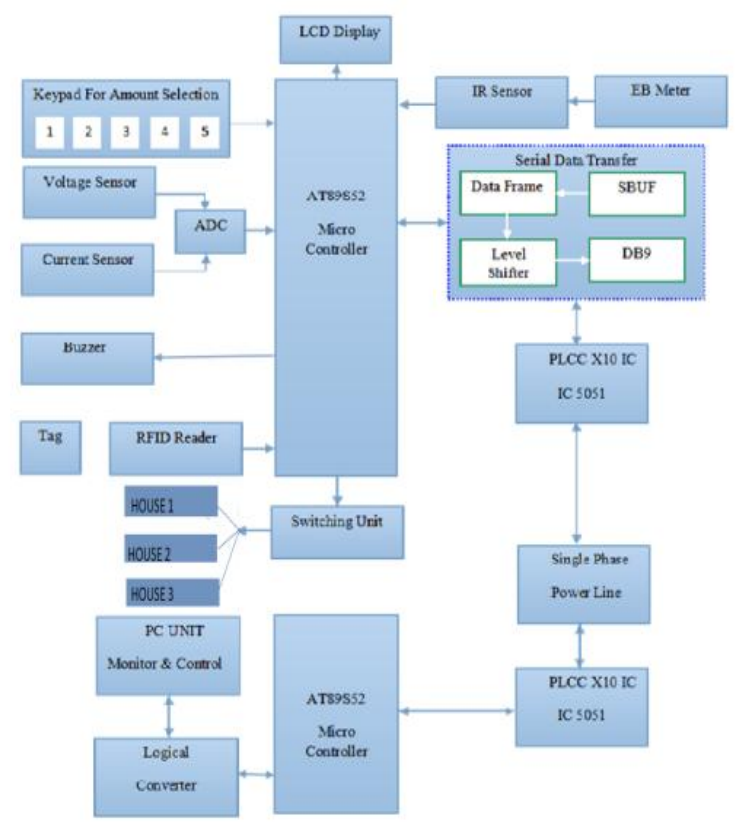

Fig. 5.1 Architecture diagram of full system.

\section{MODULE DESCRIPTION}

\subsection{AT89s52Microcontroller}

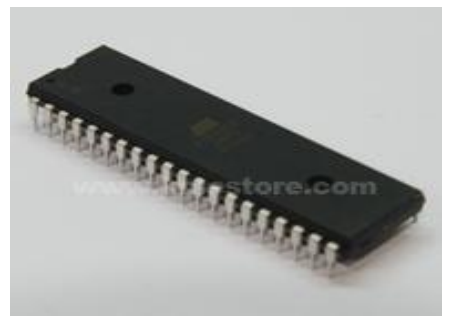

The ATMEL 8952 is a low-control, superior CMOS 8-bit microcontroller with $8 \mathrm{~K}$ bytes of in-framework programmable Blaze memory. The gadget is made utilizing Atmel's high-thickness nonvolatile memory innovation and is perfect with the Indus-attempt standard 80C51 guideline set and stick out.

\subsection{BUZZER}

A bUZZER or beeper (BUZZERS)is a flagging gadget, generally electronic, normally utilized as a part of autos, family unit apparatuses, for example, a microwave stove, or diversion appears. It most regularly comprises of various switches or sensors associated with a control unit that figures out whether and which catch was pushed or a preset time has passed, and as a rule enlightens a light on the proper catch or control board, and sounds a notice as a persistent or discontinuous humming or beeping sound.

At first this gadget depended on an electromechanical framework which was indistinguishable to an electric ringer without the metal gong (which makes the ringing commotion). Frequently these units were secured to a 
divider or roof and utilized the roof or divider as a sounding board. Another usage with some AC-associated gadgets was to execute a circuit to make the AC current into a clamor sufficiently noisy to drive an amplifier and connect this circuit to a shoddy 8-ohm speaker. These days, it is more well known to utilize an earthenware based piezoelectric sounder which makes a piercing tone. Generally these were snared to "driver" circuits which fluctuated the pitch of the sound or beat the sound on and off.

\subsection{POWERLINE CARRIER COMMUNICATION (PLCC)}

This technology has been in wide use since 1950 and was mainly used by the grid stations to transmit information at high speed. Now a days this technology is finding wide use in building/home automation as it avoids the need of extra wiring. The data collected from different sensors is transmitted on these power lines thereby also reducing the maintenance cost of the additional wiring. In some countries this technology is also provided with an Internet connection.

\section{PLC Modems/Transceiver}

PLC Transceiver is the key component of a PLCC system. It is the device which transmits $\&$ receives data to $\&$ from the power lines and acts as a hub between the power stations and our Computers/Network utilization devices. They are wired with the electrical voltage lines at home or business and work on two modes - transmit mode and receive mode. In transmit mode, they simply receive data from receiver end installed on the same network and further transmit them. In receive mode, they work the opposite way. A number of companies provide PLC transceivers and other networking devices for PLCC communication. A PLC transceiver is shown in the following image.

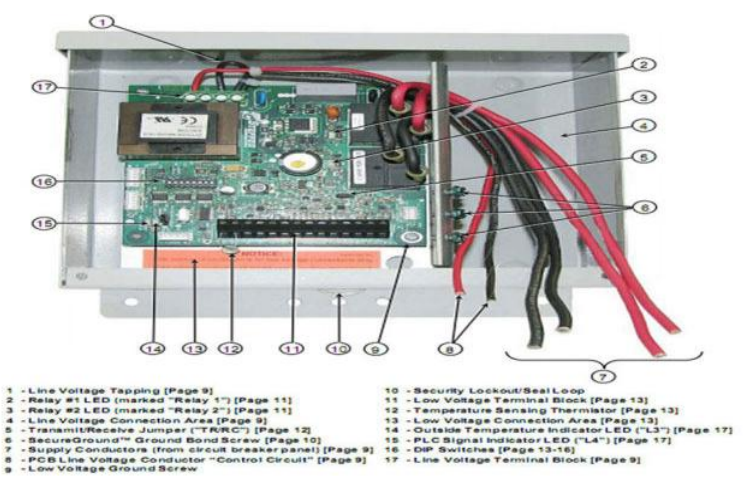

\section{X10 PROTOCOLS}

"X10" is the name given to a group of gadgets that are utilized to control lighting and apparatuses in today's Smart Homes. The main X10 items were presented as long back as 1978. From that point forward the item extend has consistently created and expanded and today X10 is perceived as a world pioneer in home computerization items.
X10 works by sending uniquely coded motions along the current power links in a building. This maintains a strategic distance from the need to introduce costly extra wiring. X10 gadgets are either transmitters, which convey the control signs or recipients, which get the flag and work the light or apparatus.

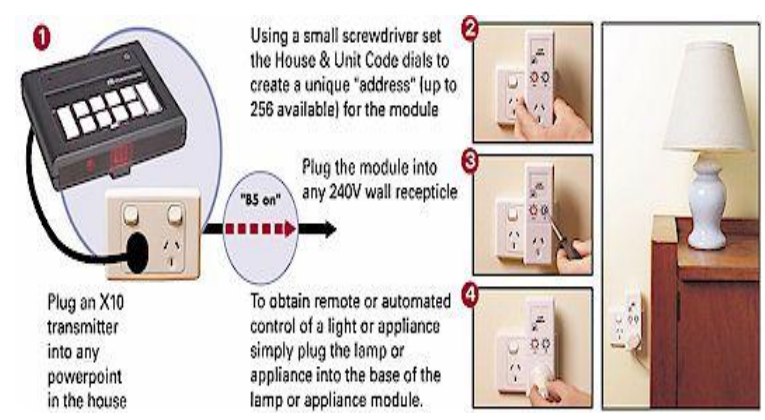

\section{Points of interest of X10}

- Because X10 utilizes the current house wiring to impart its signs it requires no additional wiring to introduce (for control of existing lights and machines).

- Installation is direct. Basic establishments can be finished by the mortgage holder. For wired-in establishment a circuit tester might be required.

- X10 has turned into an accepted worldwide standard for home automation and is utilized by numerous outsider producers.

\subsection{LCD}

Liquid crystal display is utilized to show the characters, For this various summons must be given to the LCD before contributing the required information.

LCD doesn't think about the substance (information or orders) provided to its information transport. The client needs to determine whether the substance at its information pins are information or summons. For this, if an order is inputted then a specific blend of $1 \mathrm{~s}$ must be connected to the Control lines to determine it is a Charge then again if an information is inputted at the information lines then an another mix of $1 \mathrm{~s}$ must be connected to the control lines to indicate it is Information.

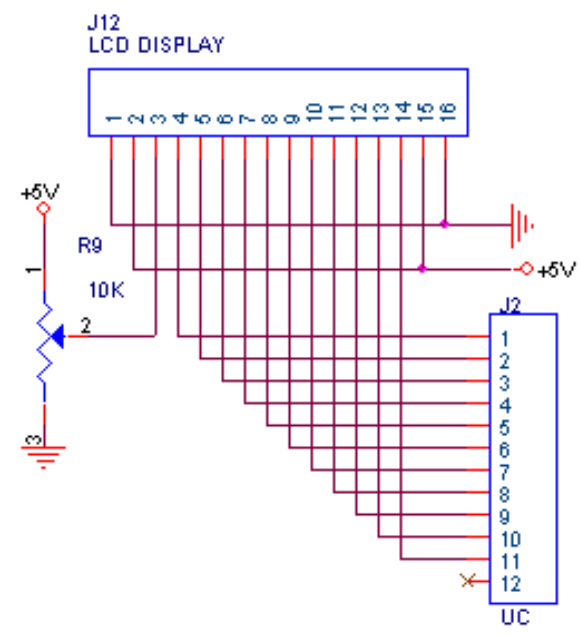




\subsection{RECTIFIER}

This rectifier is used for power supply through a circuit. A rectifier is an electrical gadget that convert alternating current into direct current or if nothing else to current with just positive esteem, a procedure known as correction. Rectifiers are utilized as segments of force supplies and as indicators of radio signs.

\subsection{IR SENSOR}

This sensor can be utilized for most indoor applications where no imperative surrounding light is available. For straightforwardness, this sensor doesn't give surrounding light insusceptibility, however a more entangled, encompassing light overlooking sensor ought to be talked about in a coming article. In any case, this sensor can be utilized to quantify the speed of protest moving at a fast, as in industry or in tachometers. In such applications, surrounding light disregarding sensor, which depend on sending $40 \mathrm{Khz}$ beat signals can't be utilized on the grounds that there are time holes between the beats where the sensor is 'visually impaired'.

The arrangement proposed doesn't contain any uncommon parts, similar to photograph diodes, photograph transistors, or IR collector ICs, just a couple if IR sensors, an Operation amp, a transistor and several resistors. In need, as the title says, a standard IR drove is utilized with the end goal of location. Because of that reality, the circuit is to a great degree straightforward, and any amateur gadgets specialist can without much of a stretch comprehend and manufacture it.

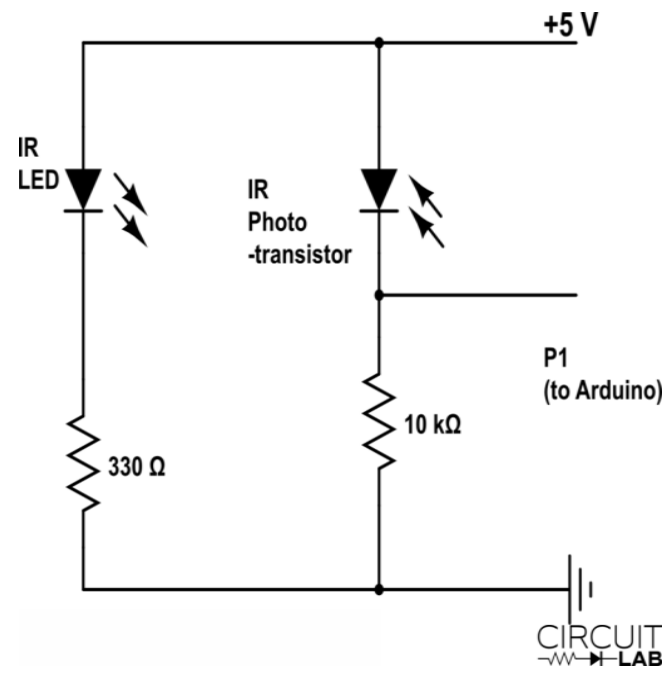

\subsection{VOLTAGE SENSOR}

The interface of different sensors to a controller like the Mind Stem GP 1.0 module normally includes either moulding or changing over voltage levels into the range the controller requires. Numerous frameworks utilize A/D converters to make the sensor esteem important in a program or information logging setup. These converters have a settled scope of voltages they can change over from with $0-5 \mathrm{~V}$ being by a long shot the most well-known.
Sensors frequently make voltages in various extents than those required by the controllers they are being interfaced to which requires the transformation of one voltage to another. This change frequently separates into a mix at least one of three types, amplification, dividing, and shifting.

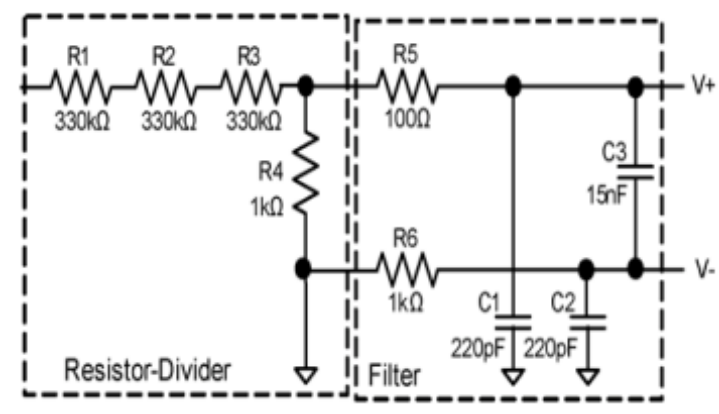

\subsection{CURRENT SENSOR}

A current sensor is a device that detects electrical current (AC or DC) in a wire, and generates a signal proportional to it.The sensed current and the output signal can be:

\section{- AC current input,}

- Analog output, which duplicates the wave shape of the sensed current

- unipolar output, which is proportional to the average or RMS value of the sensed current.

DC current input,

unipolar, with a unipolar output, which duplicates the wave shape of the sensed current.

- bipolar output, which duplicates the wave shape of the sensed current.

\subsection{RFID READER}

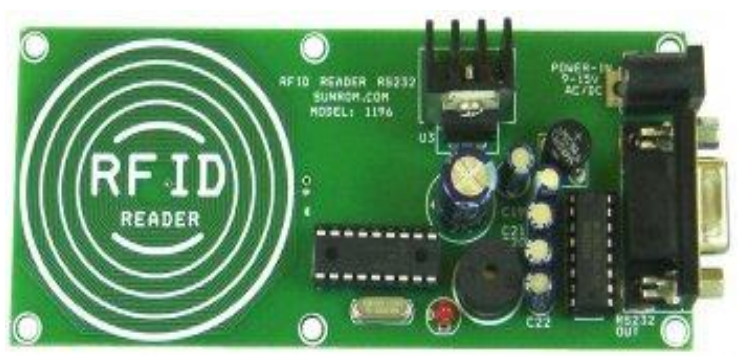

Radio-frequency identification (rfid) is the remote noncontact utilization of radio-recurrence electromagnetic fields to exchange information, for the motivations behind naturally distinguishing and following labels appended to objects. A few labels require no battery and are fueled and perused at short ranges by means of attractive fields (electromagnetic induction). Others utilize a neighborhood control source and transmit radio waves (electromagnetic radiation at radio frequencies). The tag contains electronically put away data which might be perused from up to a few meters away. Dissimilar to a standardized tag, the tag does not should be inside viewable pathway of the peruser and might be inserted in the followed object. 


\section{CONCLUSION}

This paper describes the implemented simulation framework for PLC and prepaid metering system and it also reduces the voltage fluctuation. In contrast to other studies, simulations carried out take into account all the processes in the communication layer stack.

The benefit of the approach presented is that, Prepaid meter prevents the person from heavy billing. Easy to implement in smart cities. It can reduce the man power also avoids voltage fluctuation.

The authors also believe that this simulation framework could result in an interesting tool for utilities when deploying their prepaid metering and fluctuation control solutions using PLCs. With the use of this tool, thus it is a real world application project which benefit the nation.

\section{ACKNOWLEDGEMENT}

I am exceptionally obliged to my guide for their direction and steady supervision and additionally to provide vital data in regards to the venture and likewise for their support in finishing the venture and my thanks and thanks additionally go to my partner in building up the venture and individuals who have enthusiastically bailed me out with their capacities.

\section{REFERENCES}

[1] J. Magalhaes Alves, et al. "Keen Matrices: Resource administration in essential conveyance substations, with savvy sensor systems", in Proc. of IEEE CIRED 2013, pp.1-4, 10-13 June 213.

[2] G. Accetta, D. Della Giustina, G. D'Antona, R. Faranda and S. Zanini "SmartDomoGrid: Reference Engineering and Utilize Case Investigations for a Lattice Client Communication", in Proc. 2013 IEEE ISGT-Europe, Copenhagen, Danmark, Oct. 6-9, 2013.

[3] R. Moghe, A.R. Iyer, F.C. Lambert, D.M. Divan "A Minimal effort Remote Voltage Sensor for Observing MV/HV Utility Resources", IEEE Exchanges on Keen Matrix, vol.5, no.4, pp.2002-2009, July 2014.

[4] D. Della Giustina, P. Ferrari, A. Flammini, S. Rinaldi, E. Sisinni, "Mechanization of Circulation Frameworks With IEC 61850: A First Approach Utilizing Broadband Electrical cable Correspondence", IEEE Trans. Instrumentation and Estimation, Sept., 2013, Vol. 62, N. 9, pp. 2372-2383.

[5] P. Diefenderfer, P.M. Jansson, "Control sensor applications in a heap administration organize for a private microgrid", in Proc. of 2014 IEEE SAS, pp.136,140, 18-20 Feb. 2014. 